\title{
Kinetics of shape equilibration for two dimensional islands
}

\author{
Pablo Jensen* (a), Nicolas Combe (a), Hernán Larralde (b), Jean Louis Barrat (a), Chaouqi Misbah (c) and Alberto \\ Pimpinelli (d) \\ (a) Département de Physique des Matériaux, UMR CNRS 5586, Université Claude Bernard Lyon-1, 69622 Villeurbanne \\ Cédex, FRANCE; \\ (b) Instituto de Física, Lab. de Cuernavaca, Apdo. Postal 48-3, C.P. 62251, Cuernavaca, Morelos, MEXICO \\ (c) Laboratoire de Spectrométrie Physique, Université Joseph Fourier (CNRS), Grenoble-1, B.P. 87, 38402 Saint-Martin \\ d'Hères Cédex, FRANCE \\ (d) LASMEA, Université Blaise Pascal Clermont-2, Les Cézeaux, 63177 Aubière Cédex, FRANCE
}

\begin{abstract}
We study the relaxation to equilibrium of two dimensional islands containing up to 20000 atoms by Kinetic Monte Carlo simulations. We find that the commonly assumed relaxation mechanism - curvature-driven relaxation via atom diffusion - cannot explain the results obtained at low temperatures, where the island edges consist in large facets. Specifically, our simulations show that the exponent characterizing the dependence of the equilibration time on the island size is different at high and low temperatures, in contradiction with the above cited assumptions. Instead, we propose that - at low temperatures - the relaxation is limited by the nucleation of new atomic rows on the large facets : this allows us to explain both the activation energy and the island size dependence of the equilibration time.
\end{abstract}

\section{INTRODUCTION}

There is a continued interest in the understanding, description and control of structures at the nanometer scales [1] [7]. This is partially due to technological applications of nanostructures, and partially to the fundamental interest of understanding how macroscopic concepts can (or cannot) be extrapolated down to these scales. On the technological side, a controlled preparation and conservation of these structures demands a comprehension of their time evolution, which could be rapid due to the small scales involved (typical structures contain some hundred atoms). From the fundamental point of view, it is interesting to investigate how the theoretical tools which have been developed to deal with the kinetic evolution of macroscopic objects (size larger than a micrometer) by Herring, Mullins and Nichols [9], which are based on coarse-grained, continuous equation, can be used at the nanometer scale. For example, one could wonder whether sintering of ceramic or metallic nanopowders can be analyzed with these classic tools since it is not clear that macroscopic concepts such as curvature, chemical potential, etc. should retain their relevance when dealing with structures containing only few atoms.

Here, we focus in the dynamics of equilibration of two dimensional (2D) nanocrystallites starting in an out of equilibrium shape. Mullins' theory of shape relaxation is based on the curvature being well defined. Then, two questions arise. Can one use partial differential equations to study the shape relaxation of faceted nanocrystals? Can one use them at all far from the thermodynamic limit-i.e. for small crystallites? Studying the validity of the partial differential equations approach at various length scales and temperatures is important since this formalism is also used by experimentalists to derive diffusion constants 10,11] or interpret their data 12]. Some workers 13, 14 have also used this approach as a black box to calculate the time evolution of different structures. Our study is related to one of the major problems of equilibrium surface physics : the comprehension of the relaxation of a perturbed surface profile, below its roughening temperature $T_{R}$ [15]. Above $T_{R}$, the surface (of an infinite volume crystal whose ratio surface area/volume is finite) is rough. This is equivalent to say that the step free energy vanishes identically (steps can be created at no free energy cost), and it also implies that the surface free energy is an analytic function of the local slope. Below $T_{R}$ the surface is smooth. This means that the step free energy is non-vanishing, and that the surface free energy is non-analytic. Indeed, below $T_{R}$ the surface free energy displays cusps at particular orientations, which coincide with facets in the equilibrium shape. In the thermodynamic limit, the chemical potential of a crystal is defined from the Gibbs-Thomson relation, $\mu=\Omega K$, in terms of the surface crystal curvature $K$. On facets, the curvature is ill-defined, and the chemical potential is fixed by the curved part surrounding the facet. For the relaxation of a 2D surface (the surface of a bulk crystal), it is generally believed that Mullins' treatment is correct above $T_{R}$. Below this temperature, different approaches have been proposed [16].

Molecular dynamics (MD) simulations of the coalescence of three-dimensional clusters containing roughly 1000 atoms, have shown that the relaxation kinetics is slower than predicted by Mullins' theory [17]. However, MD simulations are still limited in computation time (no more than $\sim 10 \mathrm{~ns}$ ) and it is therefore difficult to follow the coalescence at temperatures not too close to the melting temperature (see also [4]). An alternative method consists in using Kinetic Monte Carlo (KMC) simulations which allow an incomparable larger range of time studies. As a first attempt, we have chosen to study a two-dimensional (2D) system, namely the relaxation ki- 
netics of 2D faceted islands supported on a triangular lattice.

The basic idea is the following : we start with a island with a shape clearly not an equilibrium one (e.g. with an $x$ side 10 times longer than the $y$ side) and anneal it at a given temperature. Indeed, the perimeter free energy dictates the equilibrium shape, which is the one that minimizes the island free energy at given volume (here we expect hexagonal shapes because of the lattice geometry). We assume that the island has relaxed when the aspect ratio (defined as the ratio of the $x$ and $y$ gyration radii, $\left.\alpha=\rho_{x} / \rho_{y}\right)$ becomes 1 . We then monitor the kinetics of the relaxation process, which depends on the precise pathway chosen by the island to change its shape. We are particularly interested in the influence of facets in the relaxation kinetics. For this reason, we perform simulations at several temperatures: at high temperatures, where the islands contour is clearly rough and only small facets can be distinguished; at low temperatures, where clear-cut and persistent facets are apparent. This point is quite delicate, because a $2 \mathrm{D}$ object-whose contour is a line, not a surface - is not expected to show facets at any temperature in the thermodynamic limit. Indeed, a line is rough at all non-zero temperatures, and the line tension $\gamma\left(\mathrm{Jm}^{-1}\right)$ is analytic for all orientations 15. However, facets do appear at $T=0$, and at low $T$ one would expect the persistence length of a facet (the average distance between kinks) to be quite large. If it is larger than the island side, then facets are indeed expected, as we observe. In other words, creating a kink costs a finite energy, which is always compensated by the entropy gain when the length of the line goes to infinity (cf. Landau's argument for the non-existence of phase transitions in 1D). As long as the line is finite, facets occur. One could then guess that they affect the kinetics, even for $T>T_{R}=0$.

Our main conclusion is that equilibration of an island's shape is a non-universal process, in which the time evolution of the shape does not obey scaling, while it strongly depends on temperature, and thus on system-dependent features like the energy scale $E$. Scaling relations can be found for the relaxation (or equilibration) time, as a function of temperature and island size. Indeed, two regimes with two different scaling forms are born out by the simulations, at high and low $T$, respectively. We tentatively attribute these two regimes to the absence and presence of facets, respectively.

\section{MONTE CARLO AND PARTIAL DIFFERENTIAL EQUATION APPROACH}

\section{A. Kinetic Monte Carlo simulations}

We perform "standard" kinetic Monte Carlo (KMC) simulations on a triangular lattice. We assume that the potential energy of an atom is proportional to its number of neighbors, and that the kinetic barrier for diffusion is also proportional to the number of initial neighbors, regardless of the final number of neighbors, i.e. after the jump (see Fig. 11). This is of course a huge simplification, which is however aimed here at describing the global evolution of a model island. In other words, we do not wish to study any particular system but rather to investigate properties which should not depend on the details of atom-atom interaction. Therefore, we use a simple kinetic model containing as few parameters as possible (only one, the ratio $E / k_{B} T$ where $E$ sets the energy scale ( $E=0.1 \mathrm{eV}$ throughout the paper), $k_{B}$ is the Boltzmann constant and $T$ the absolute temperature). Comparing with recent ab-initio calculations [8] for the $\mathrm{Al}(111)$ surface, we note that our one-barrier assumption does give the good order of magnitude of the relative jump frequencies for the different hopping processes of interest here. We also exclude any explicit "Ehrlich-Schwoebel" barrier [18 for atoms hopping around corners, although the occurrence of atoms with a single neighbor is treated in a special way (see below). The kinetic barriers for some jumps are shown in Fig. 2. In the same spirit as ours, a similar but slightly more complicated model has been used recently by Metiu's group with the scope of obtaining system-independent information on island diffusion on a surface: these authors investigate the existence of a "universal" size dependence of the island diffusion constant. They conclude that such universality is not observed, and we observe a similar phenomenon for island equilibration.

The time evolution of the island shape is obtained by the following algorithm. We first calculate the following quantities : $\delta_{1}=\exp \left[-E /\left(10 k_{B} T\right)\right], \delta_{2}=$ $\exp \left[-2 E /\left(k_{B} T\right)\right]$ and $\delta_{3}=\exp \left[-3 E /\left(k_{B} T\right)\right]$ which represent the relative weights for the jump probabilities for atoms with respectively 1, 2 or 3 neighbors (atoms with more neighbors simply do not move : see an explanation of the precise forms of the different $\delta_{i}$ below). Then, in each iteration, we calculate the probability to move an atom with $i$ neighbors as :

$$
p_{i}=\frac{(6-i) n_{i} \delta_{i}}{\sum_{i=1}^{3}(6-i) n_{i} \delta_{i}}
$$

where $n_{i}$ is the total number of atoms having $i$ neighbors. We choose randomly one of the atoms with the appropriate number of neighbors and move it in a random direction. The time is increased at each iteration by

$$
d t=\left(\nu_{0} \sum_{i=1}^{3}(6-i) n_{i} \delta_{i}\right)^{-1}
$$

where $\nu_{0}$ is a Debye frequency (we have taken $\nu_{0}=$ $\left.10^{13} s^{-1}\right)$. To check that the law of detailed balance is satisfied, one can refer to Fig. 11: the probability for an atom to jump from a site having $n$ neighbors to a site having $p$ 
neighbors is $\delta_{n}$ while the opposite transition has a probability $\delta_{p}$. Their ratio is $\delta_{n} / \delta_{n}=\exp \left(-E /\left(k_{B} T\right)(n-p)\right)$, i.e. equal to the energy difference of the initial and final configurations, as required by the law of detailed balance (the particular case when $n$ or $p$ is equal to 1 can be analyzed in the same way). This algorithm is very fast 19 21 since all iterations contribute to the evolution (there are no rejected moves). One peculiarity of this model is the treatment of atoms having one single neighbor: $\delta_{1}$ is much larger than what one could expect from the general rule $\delta_{n}=\exp \left[-n E /\left(k_{B} T\right)\right]$ : indeed, we let $\delta_{1}=\exp \left[-E /\left(10 k_{B} T\right)\right]$ instead of $\delta_{1}=\exp \left[-E /\left(k_{B} T\right)\right]$. This is to ensure that singly-bonded atoms, which are in some sense in a "transition state", rapidly go into some physically reasonable position, i.e. one having 2 or more neighbors. Note also that detachment of atoms from the islands is forbidden here: equilibration is only due to mass transport along the island contour. This is clearly different from Ostwald ripening where islands evolve in equilibrium with a two-dimensional adatom gas. We note that a recent experimental study by Stoldt et al. 22 has shown that supported Ag two dimensional islands do indeed relax via atomic diffusion on the island edge, without significant contribution from exchange with a twodimensional adatom gas. A last remark on the algorithm used here : we do not allow atoms having more than 3 neighbors to move. In some sense, they have an infinite potential energy. Since our potential energy is not very realistic anyway, this hypothesis allows to simplify and accelerate the simulations. The key point is that our results are particularly interesting at low temperatures, where including the possibility for atoms with 4 neighbors to jump would make no significant difference in the kinetic evolution of the island because at these temperatures their jumping is vanishingly small.

\section{B. Partial Differential Equation approach}

A complementary approach at predicting the evolution of a crystal shape at a temperature higher than the roughening transition, consists in coarse-graining the crystal profile, in order to treat it as a smooth function $h(x, t)$, and in writing down its time evolution in the form of a partial differential equation, whose form depends on the physical situation of interest. The situation when matter transport is assured by adatom diffusion along the surface, has been originally considered by Mullins and coworkers [9], for studying small deformations of an infinite planar surface. The case of a finite, closed "surface" - the island contour — is somewhat more subtle, and we give the derivation in some detail. The evolution equation has in general then the form of an equation for $s$ the curvilinear coordinate or arclength. On purely geometrical grounds, it can be shown [23] that this equation can be written as an evolution equation for the curvature $K(s, t)$, of the form

$$
\frac{\partial K}{\partial t}=-\left[\frac{\partial^{2}}{\partial s^{2}}+K^{2}\right] v_{n}-\frac{\partial K}{\partial s} \int \mathrm{d} s^{\prime} K v_{n}
$$

where $v_{n}$ is the normal velocity of the interface. The latter is fixed by the physics of the problem. In our case, when edge diffusion is the relevant physical process determining the relaxation of the shape, the equation for $v_{n}$ must have the form of a conservation equation for the island area

$$
v_{n}=-\frac{\partial \mathbf{j}}{\partial s}
$$

The edge diffusion current $\mathbf{j}$ is given by the gradient of the local excess chemical potential as in Fick's law :

$$
\mathbf{j}=-\frac{\tilde{D}}{k_{B} T} \frac{\partial \Delta \mu}{\partial s}
$$

where $\tilde{D}$ is a (collective) perimeter diffusion coefficient.

The excess chemical potential is in turn related to the local curvature $K(s)$ through the Gibbs-Thomson relation:

$$
\Delta \mu=-\gamma a^{2} K
$$

where $\gamma$ is the line tension (that for simplicity is assumed isotropic here), and $a$ the depth of the outer layer within which mass transport takes place (one lattice spacing in our case). Eqs. (2.5) and (2.6) yield

$$
\mathbf{j}=-\frac{\tilde{D} \gamma a}{k_{B} T} \frac{\partial K}{\partial s}
$$

and the corresponding evolution equation (2.4) is given, in our two-dimensional situation, by:

$$
v_{n}=\frac{\tilde{D} \gamma a^{4}}{k_{B} T} \frac{\partial^{2} K}{\partial s^{2}}
$$

Equations (2.3) and (2.8) must be solved simultaneously for the island shape: the results will be shown in section IIID. The equilibrium solution $v_{n}=0$ obviously is a constant-curvature shape, that is a circle. Also, note that equations (2.3) and (2.8) are invariant by a rescaling $s \rightarrow \lambda s, t \rightarrow \lambda^{4} t$ [9], so that the equilibration time of a deformed island of size $L$ is expected to be proportional to $L^{4}$ or $N^{2}$ where $N$ is the number of atoms in the island.

\section{MONTE CARLO SIMULATION RESULTS}

To study the influence of the facets on the coalescence kinetics, it is interesting to study the time evolution of the aspect ratio and the size, as well as the temperature dependence of the equilibration time $t_{e q}$. We recall that $t_{e q}$ is defined as the time needed for the island to reach its equilibrium shape. In practice, we take $t_{e q}$ as the first time when the aspect ratio $\alpha$ defined above becomes less than 1. Each point is the average of several runs (up to 200 for the smallest islands). 


\section{A. Island morphology}

Fig. 3a shows the time evolution of the perimeter of a 6250 atoms island at $500 \mathrm{~K}\left(E / k_{B} T=2.3\right)$. It is clear that the shape evolution occurs with rough island borders.

Fig. 3b shows the time evolution of the perimeter of an island containing 6250 atoms at $83 \mathrm{~K}\left(E / k_{B} T=14\right)$. At this temperature facets are apparent throughout the evolution.

A more precise comparison of the presence of facets at the two temperatures studied above is given in Fig. 4. It is apparent that facets are present at $83 \mathrm{~K}$, in contrast to the high rugosity observed at $500 \mathrm{~K}$.

\section{B. Dependence of the equilibration time on island size}

The continuous analysis of section II B predicts $t_{e q} \sim$ $N^{2}$ as a function of the number of atoms $N$ inside the island, for any temperature. Indeed, the numerical solution of the full, non-linear equations (2.3) and 2.8 appears to agree with this prediction. Fig. 5a shows the size dependence of $t_{e q}$ for different temperatures as given by the simulations. The simulation results agree with $t_{e q} \sim N^{2}$ only at high temperatures. Below 250 $\mathrm{K}$, it is clear that $t_{e q}$ increases slower than $N^{2}$, and the lower the temperature, the smaller the exponent. One can also notice (Fig. 5b) that the local exponent for low temperatures approaches 1 for the highest island sizes. This is analyzed in Sec. [V] where we give an attempt at deriving a scaling relation describing the two regimes. It should also be noted that extrapolating the different curves for very high values of the island size leads to an apparently absurd conclusion : very large islands do equilibrate faster at lower temperatures. This is a immediate consequence of the higher size exponents found for the highest temperatures. To avoid a paradox, we must admit that there exist a crossover from high to low temperature behavior for a given size that depends on the temperature. Therefore, even at $83 \mathrm{~K}$ (highest curve), for large enough islands, one should recover the $t_{e q} \sim N^{2}$ regime. The scaling analysis presented below explains this crossover.

\section{Dependence of the equilibration time on temperature}

Fig. 6a shows that $t_{e q}$ rapidly increases as temperature decreases, in roughly the same way for all the island sizes. The equilibration time is not exactly a thermally activated quantity, since there is a clear curvature in its Arrhenius plots, as shown in Fig. 6 b: the local activation energies increase from roughly $0.3 \mathrm{eV}$ at high $T$ to $0.4 \mathrm{eV}$ at low temperature. This represents respectively
3 and 4 times the energy needed to break a single bond. A tentative interpretation of these values is given below in Sec. IV.

\section{Precise kinetics of the relaxation}

We have seen that the size dependence of the equilibration time obeys the scaling predicted by the linearized equations only at high temperatures. It is interesting to check whether the full solution of equations (2.3) and (2.8) agrees with the high-temperature behavior of the $\mathrm{MC}$ simulations, when no facets are apparent, and the island looks rough and rather isotropic. Fig. [7, where the aspect ratio is plotted as a function of the reduced time $t / t_{e q}$, shows that no agreement is found, at any of the studied temperatures. Indeed, this is a posteriori not surprising, since the MC results do not seem to obey any scaling relation, or maybe only at high temperature, and it is then obvious that the "universal" description given by continuous equations does not apply. It is nevertheless a little surprising that the continuous description, which agrees with simulations in the case of a planar surface above the roughening temperature, does not seem to set a limiting behavior valid for very large sizes $(N \rightarrow \infty)$, nor to provide the scaling form which seems to appear in the simulation results at high temperatures.

We can think of (at least) three explanations of this observation. First, it could be argued that this is an effect of the edge tension. Indeed, in writing the constitutive equation (2.8) we have assumed that $\gamma$ is isotropic. This is clearly not the case in the simulations since we take a triangular lattice, and the energy of the facets depends on the orientation. We are currently performing a numerical integration of equations (2.3) and (2.8) including anisotropic line tension to further investigate this point.

Second, one could question the adequacy of the continuous treatment to describe the detailed path to equilibrium, even at high temperatures, for small clusters. Indeed, one could argue that the macroscopic concepts of curvature, chemical potential, etc. are not adapted to deal with nanometric objects containing few (less than 10000) particles.

Finally, it is possible that the simulations are not adapted to agree with the continuous theory, in the sense that our interatomic potential is too crude to give a reasonable kinetic path to equilibrium. It is clear that the assumption that the transition probability depends only on the initial state is not generally correct. As has been argued above (section IIA), one would expect such a rough potential to reproduce a universal exponent (as is observed for $t_{e q} \propto N^{2}$ at high temperatures) but not necessarily a detailed time evolution, if the latter is nonuniversal. 


\section{A SCALING ARGUMENT}

Our MC results show that two different regimes - at low and high temperatures - can be identified. At first sight, this is surprising since the only occurrence that could separate high from low temperatures is the roughening transition, which, strictly speaking, takes place at $T=0 \mathrm{~K}$ in 2 dimensions - that is, for a one-dimensional "surface". However, facets do not disappear suddenly as the temperature is raised from $T=0$. Indeed, a "persistence length" of facets can be defined as the equilibrium value of the distance between kinks along the step edge. At equilibrium and at low temperatures, we can consider an "ideal gas" of kinks, whose density (number of kinks per unit length) is given by the formula 24.

$$
n_{\text {kink }}=2 \exp (-\beta W) / a
$$

where $W$ is the kink creation energy and $a$ the lattice spacing. Indeed, to form kinks one has to take an atom out of the step edge, and to place it anywhere along the step. On a triangular lattice, in doing so one looses 4 nearest-neighbours bonds, and gains 2. A net balance of 2 broken bonds results. In the process, 4 kinks have been created (each atom counts for 2 kinks), so that the energy cost per kink is

$$
W=E / 2 \text {. }
$$

The factor of 2 in (4.1) comes from the fact that kinks always appear and disappear in pairs - in other words two types of kinks, positive and negative exist, of equal number. Then, the equilibrium distance between kinks is

$$
\ell_{0}=a \exp (\beta E / 2) / 2
$$

which diverges as $T$ goes to 0 . At a given temperature, a step looks straight (free from kinks) over lengths of the order of $\ell_{0}$. An island is thus bound to look faceted as long as $\ell_{0}$ is larger than the island linear size $L$. Then, the approximate equality

$$
\exp \left(E /\left(2 k_{B} T_{c}\right) / 2 \approx L_{c} / a\right.
$$

gives the "crossover size" $L_{c}$ (at fixed temperature) or the "crossover temperature" $T_{c}$ (at fixed size) for the crossover between the high (rough) and low (faceted) temperature regimes. A comparison with Fig.(5b), where the high temperature regime corresponds to $t_{e q} \sim N^{2}$, while the low temperature regime corresponds to $t_{e q} \sim$ $N$, shows that this criterion is not too bad: the formula predicts a crossover temperature for an island of size $N=500(L \approx 22)$ of approximately $240 \mathrm{~K}$, in good agreement with the simulation data. Note that for smaller sizes than about $N \approx 100$ the simulations do not show a well defined low temperature behaviour. We attribute this to the large importance of geometric kinks (imposed by the fact that the step closes on itself) over thermal kinks for these small sizes. Indeed, we claim that the low temperature regime is ruled by the equilibration of the spatial distribution of thermal kinks: the initial shape creates a strongly inhomogeneous distribution of these kinks, which then diffuse to achieve spatial uniformity, and thus equilibrium. Kinks diffuse by emitting atoms, so that we conclude that atom emission from kinks is the limiting kinetic step determining the low temperature behaviour of $t_{e q}$.

Based on this assumption, we can give a scaling argument that reproduces well the observed $t_{e q}$ as a function of $N$ and $T$. The argument is similar to that used by Bales and Zangwill [25] and Pimpinelli et al. 26] to discuss step roughening and smoothening during growth and at equilibrium. Indeed, it amounts to performing a linear stability analysis, and computing the relaxation time of a perturbation of given amplitude and wavelength. For sake of clarity, we start from the discussion of the high temperature behaviour, which is well known from Mullins work.

Physically, we assume that there are two ingredients determining the relaxation : the thermodynamical "force" which drives the relaxation (here, the excess curvature) and second, the kinetic factors which determine the rate of the equilibration. At all temperatures, curvature effects are relevant, but we assume that the kinetics change due to the presence of facets (or, equivalently, to the low concentration of kinks). The transition takes place, as stated above, when $\ell_{0} \approx L_{c}$. Here is the mathematical translation of this idea.

\section{A. High temperatures}

Let $\delta_{q}$ be the amplitude of a perturbation of wave vector $q$ of the island perimeter with respect to the equilibrium shape. The curvature effect (Gibbs-Thomson) opposes the increase of the deformation. The rate of decrease depends on the appropriate kinetic process which limits transport of matter from high to low chemical potential regions. Let $n_{e q}$ be the equilibrium atom density along a reference island edge with the equilibrium shape. Then, at high temperature, a deformation of local curvature $K$ results in an excess chemical potential $\Delta \mu \sim$ $\gamma q^{2} \delta_{q}$ as in equation (2.6). In turn, this creates an excess atom density $n_{e x c}=n_{e q} \exp \left[\Delta \mu /\left(k_{B} T\right)\right] \approx n_{e q}\left[1+\Gamma q^{2} \delta_{q}\right]$, where $\Gamma=\gamma /\left(k_{B} T\right)$. Then, edge atoms flow away from the deformation, whose amplitude decreases at a rate proportional to the divergence of the mass current :

$$
\dot{\delta}_{q} \approx-\frac{1}{\tau^{*}} \nabla^{2}\left(n_{e x c}-n_{e q}\right) \approx-\frac{n_{e q}}{\tau^{*}} q^{2} \times \Gamma q^{2} \delta_{q}
$$

where $\tau^{*}$ is the typical timescale of the appropriate kinetic process which is responsible of matter transport.

A more detailed justification of this expression can be found in Mullins [], Bales and Zangwill [25] and Pimpinelli et al. 26. 
Defining the equilibration time $t_{e q}$ by writing $\dot{\delta}_{q=1 / L} \approx$ $-\delta_{q=1 / L} / t_{e q}$ gives

$$
t_{e q} \approx L^{4} \frac{\tau^{*}}{n_{e q} \Gamma} \approx N^{2} \frac{\tau^{*}}{n_{e q} \Gamma} .
$$

Mullins equation is recovered if one assumes that atom edge diffusion limits the kinetics, so that

$$
\frac{1}{\tau^{*}} \approx D
$$

The atom equilibrium density can be obtained from the detailed balance at the kinks: $D n_{e q}=\nu_{k i n k}$, where $\nu_{k i n k}=\nu_{0} \exp \left[-3 E /\left(k_{b} T\right)\right]$ is the rate of atom emission from kinks and $D n_{\text {eq }}$ is the atom flux to the kinks [27,28]. Thus,

$$
n_{e q}=\nu_{0} / D_{0} \exp \left[-E /\left(k_{b} T\right)\right] .
$$

Inserting (4.7) and (4.8) in Eq. 4.6 yields, in the limit of high temperatures,

$$
t_{e q} \approx \frac{1}{\Gamma \nu_{0}} N^{2} \exp \left[3 E /\left(k_{b} T\right)\right]
$$

This prediction reproduces the $t_{e q} \sim N^{2}$ scaling of the continuum theory, and it is in very good agreement with the simulation results obtained at high temperatures both for the temperature dependence and for the size dependence (Fig. 5. Indeed, at high temperature the equilibration time shows an activation energy of approximately $3 E$ (Figs. 6), and $t_{e q}$ behaves $\sim N^{2}$ in this regime.

\section{B. Low temperatures}

The low temperature regime sets in, for a given crystal size, when the equilibrium distance between kinks becomes of the order of the linear size of the crystal, and straight step portions appear. The (thermal) kink density then becomes a relevant concept. When the crystal is deformed from the equilibrium shape, the kink density is increased where the facets are shrunk, and decreased where they are streched. On removing the constraint, the kink density tends to equilibrium and seeks spatial uniformity. If the equilibrium facet size is $L \approx N^{1 / 2}$, and a shape deformation of order $\delta \ell$ is introduced, the kink density unbalance is approximately $\delta \ell / L^{2}$. Then, the perturbation relaxes as

$$
(\dot{\delta} \ell) \approx-\frac{1}{\tau^{* *}} \times \frac{1}{\mathrm{E}^{2}} \delta \ell \approx-\frac{1}{\tau^{* *}} \frac{1}{N} \delta \ell .
$$

The relaxation proceeds by moving a whole row of atoms from a short to a long facet; diffusion is fast on facets, and the process is limited by nucleation of the new row, that is, by the rate of atom encounters $D n_{e q}^{2}$. Then,

$$
\frac{1}{\tau^{* *}} \approx D n_{e q}^{2}=\nu_{0}^{2} / D_{0} \exp \left[-4 E /\left(k_{B} T\right)\right]
$$

Inserting (4.11) and (4.8) in Eq. 4.10 yields, at low temperatures,

$$
t_{e q} \approx \frac{D_{0}}{\nu_{0}^{2}} N \exp \left[4 E /\left(k_{B} T\right)\right]
$$

Again, the activation energy predicted here is in good agreement with the low temperature limit observed in the simulations (Fig. 5). The scaling $t_{e q} \sim N$ is less clearly seen in the simulations (Figs. 6). However, the simulations show that the lower the temperature, the lower the size exponent, and if $N$ is not too small, $t_{e q} \sim N$ is consistent with our results. When $N$ is smaller than about $100, t_{e q}$ seems to increase faster than linearly. At such small sizes, facets are always very short, and it is likely that an intermediate behaviour between mass transport and facet nucleation rules the relaxation.

\section{Discussion}

The scaling argument we propose nicely reproduces the results of our simulations and leads to a reasonable physical picture of the equilibration, consistent with the observed morphologies and kinetics (presence of facets, rapid completion of atomic rows ...).

Even more, our results can be used to estimate the behaviour of the diffusion coefficient of a cluster as a function of the cluster size $N \approx L^{2}$, by means of another scaling argument. In order to diffuse over a length $\ell$, a number of atoms of order $\ell L$ have to be transferred from one side of the island to the opposite side. The time needed to do this is of the order of the time $t_{e q}(L)$ needed to equilibrate a fluctuation of linear size $L$ and mean square amplitude $\ell^{2} \approx L /(\beta \gamma)$ [15]. Therefore, from the knowledge of $t_{e q}(L)$ we can know the diffusion coefficient $D(N)$ from the Einstein relation

$$
D(N) t_{e q}(N) \approx \ell^{2} \sim N^{1 / 2} .
$$

If we assume that our high-temperature result $t_{e q}(N) \sim N^{2}$ holds, we find

$$
D(N) \sim N^{-3 / 2} .
$$

If we assume that our low-temperature result $t_{e q}(N) \sim$ $N$ holds, we find

$$
D(N) \sim N^{-1 / 2} .
$$

Equations (4.14) and (4.15) can be compared with the results of the simulations of island diffusion of Metiu and coworkers': on a (001)-type substrate they find that the size-dependent diffusion constant $D(N)$ of $2 \mathrm{D}$ islands varies as $D(N) \sim 1 / N^{1.52}$ at high temperature, and as $D(N) \sim 1 / N^{0.62}$ at low temperature [29]. Of course, 
different equilibration processes would lead to different $t_{e q}(N)$ s and then to different behaviours for $D(N)$. This might explain the different results obtained by Bogicevic et al. [30 for islands diffusing on a (111) substrate but with energy barriers for the jumps different from those assumed here.

\section{SUMMARY, PERSPECTIVES}

The relaxation to equilibrium of $2 \mathrm{D}$ islands containing up to 20000 atoms shows unexpected features. Our results show that there is no "universal" size exponent for island equilibration, a result similar to that found by Metiu's group for island diffusion [30]. We are now studying the case of $3 \mathrm{D}$ clusters to check both the scaling of the equilibration time with the size of the particle and the precise kinetic path followed to reach equilibrium. This is done by KMC simulations and an analytical approach.

* e-mail address : jensen@dpm.univ-lyon1.fr

[1] M. Lagally, Physics Today 46(11), 24 (1993) and references therein; H. Gleiter, Nanostructured Materials 11 (1992); Z. Zhang and M. G. Lagally, Science 276, 377 (1997)

$[2]$

http://www.msel.nist.gov/structure/metallurgy/techactv95/ http://nanoweb.mit.edu/ http://www.eas.asu.edu/ nano/nano2.html

[3] M. Flüelli, P. A. Buffat, and J. P. Borel, Surf. Sci. 202, 343 (1988).

[4] X. Yu and P.M. Duxbury, Phys. Rev B 52, 2102 (1995).

[5] H. Shao, S. Liu and H.Metiu, Phys Rev B 517827 (1995)

[6] H. Zhu and R.S. Averback, Phil. Mag. Lett. 73, 27 (1996).

[7] A. Perez et al, J. of Physics D 30, 1 (1997).

[8] A. Bogicevic, J. Strömquist and B. Lundqvist, Phys. Rev. Lett. 81, 637 (1998)

[9] C. Herring, Physics of Powder Metallurgy (McGrawHill Book New York, Company, Inc. 1951) Ed. W. E. Kingston; Phys Rev 8287 (1951); C. Herring, Phys Rev 8287 (1951); W.W. Mullins, J. Appl. Phys. 28, 333 (1957) and 30, 77 (1959); F.A. Nichols and W.W. Mullins, J. Appl. Phys., 36, 1826 (1965); F.A. Nichols, J. Appl. Phys. 37, 2805 (1966).

[10] M. Drechsler et al. Journal de Physique 50, Colloque C8, 223 (1989)

[11] H. P. Bonzel and E. E. Latta, Surf. Sci. 76, 275 (1978)

[12] G. Jeffers, M. A. Dubson and P. M. Duxbury, J. Appl. Phys. 75, 5016 (1994)

[13] N. Olivi-Tran, R. Thouy and R. Jullien, J. Phys I France
6557 (1996); R. Thouy, N. Olivi-Tran and R. Jullien, Phys. Rev B 56, 5321 (1997)

[14] J. Eggers, Phys Rev Lett. 802634 (1998)

[15] J. Villain and A. Pimpinelli Physique de la Croissance Cristalline (Eyrolles, 1995); A. Pimpinelli and J. Villain Physics of Crystal Growth (Cambridge University Press, 1998)

[16] F. Lanon and J. Villain, in Kinetics of Ordering and Growth at Surfaces M.G. Lagally ed. (Plenum, New York) p. 369 (1990)

[17] L. Lewis, J. L. Barrat and P. Jensen, Phys Rev B 56 2248 (1997)

[18] R. L. Schwoebel, J. Appl. Phys. 40, 614 (1969); R. L. Schwoebel and E. J. Shipsey, J. Appl. Phys. 37, 3682 (1966); J. Villain, J. Physique I 1, 19 (1991)

[19] A. B. Bortz, M. H. Kalos and J. L. Lebowitz, J. Comp. Phys. 1710 (1975)

[20] H.C. Kang, W.H.Weinberg, J.Chem.Phys. 90(5), 2824 (1989).

[21] Y.T.Lu, H.Metiu, Surf. Science, 245150 (1991).

[22] C. R. Stoldt, A. M. Cadilhe, C. J. Jenks, J. M. Wen, J. W. Evans and P. A. Thiel, Phys Rev Lett. 812950 (1998)

[23] C. Misbah and A. Valance, unpublished

[24] W. K. Burton, N. Cabrera, and F. Frank, Phil. Trans. Roy. Soc. 243, 299 (1951)

[25] G. S. Bales and A. Zangwill, Phys. Rev. B 41, 5500 (1990).

[26] A. Pimpinelli, J. Villain, D. E. Wolf, J. J. Métois, J. C. Heyraud, I. Elkinani and G. Uimin, Surf. Sci. 295, 143 (1993); A. Pimpinelli, I. Elkinani, A. Karma, C. Misbah and J. Villain, J. Phys.: Condens. Matter 6, 2661 (1994)

[27] G.S. Bales and D.C. Chrzan, Phys. Rev. B 50, 6057 (1994)

[28] P. Jensen, H. Larralde and A. Pimpinelli, Phys. Rev. B $\mathbf{5 5 , 2 5 5 6 - ( 1 9 9 7 )}$

glostrug ettml (private communication) : more details to be found in Metiu, Mattsson and Mills, MRS Symposia (1998), to be published

[30] A. Bogicevic, S. Liu, J. Jacobsen, B. Lundqvist and H.Metiu, Phys Rev B 57 R9459 (1998) 


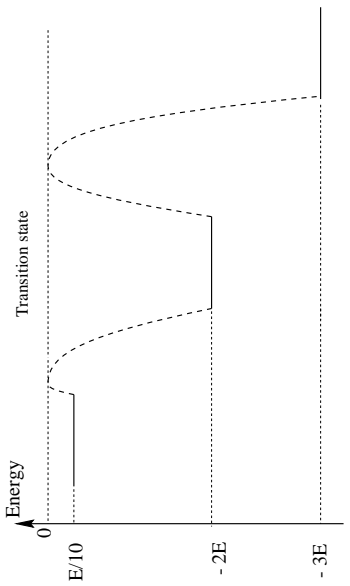

FIG. 1. Potential energy of an atom diffusing along the island edge when it has 1 (energy : -E/10), 2 (energy : -2E) or 3 (energy : $-3 \mathrm{E}$ ) neighbors. The atom energy is supposed to depend only on the number of first neighbors and the transition state is assumed to lie at the same energy for all jumps, taken here as the origin of energies. As a consequence, the energy barriers for diffusion are equal to the potential energies of the atoms, ensuring detailed balance (see text).

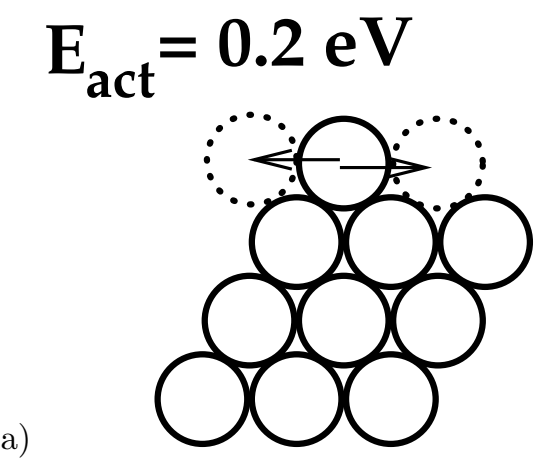

(b)
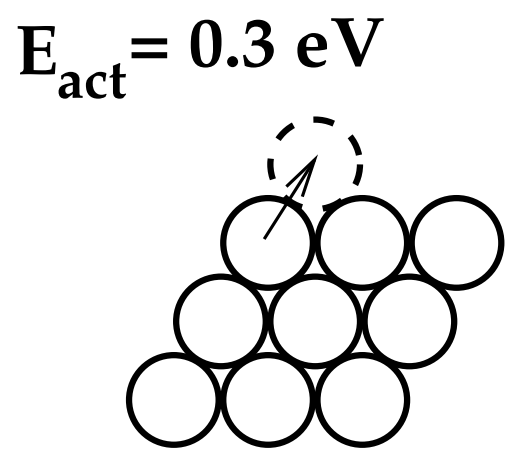

FIG. 2. Examples of activation energies for different atomic jumps on the island edge. Note that detachment of atoms from the island is explicitly forbidden in the simulations.

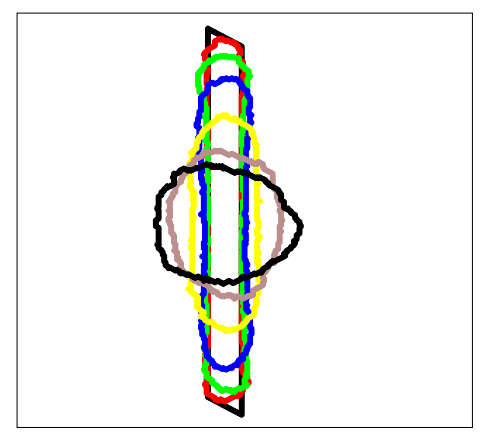

(a)

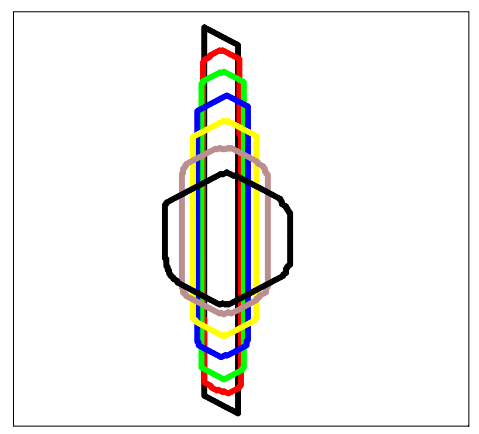

(b)

FIG. 3. Time evolution of islands containing 6250 atoms at $500 \mathrm{~K}$ (a) and $83 \mathrm{~K}(\mathrm{~b})$. The initial state corresponds to the most elongated configuration.

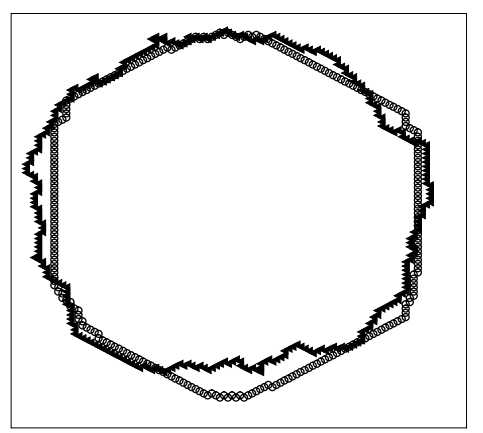

FIG. 4. Large facets are present at $83 \mathrm{~K}$ (open circles) in contrast with the rugosity observed at $500 \mathrm{~K}$ (filled triangles) 
(a)

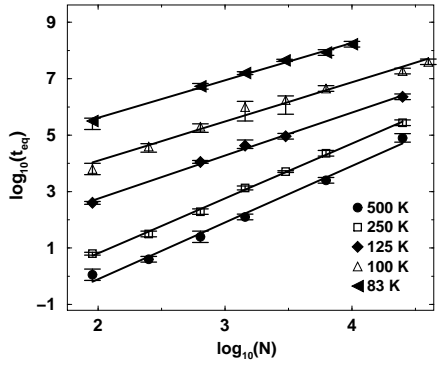

(b)

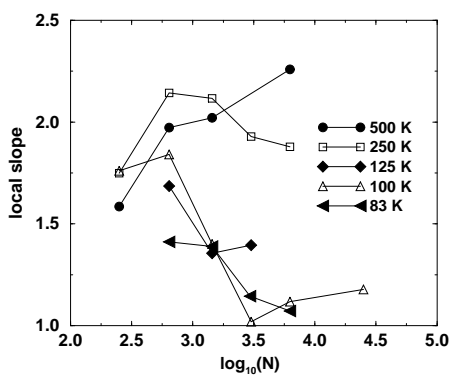

FIG. 5. (a) Size dependence of $t_{e q}$ for different temperatures and

local slope of the size dependence (obtained as the discrete derivative of the $t_{e q}$ vs $N$ curve given in (a) : for point i, it is $\left(\log \left(t_{e q}(i+1)\right)-\log \left(t_{e q}(i-1)\right)\right) /(\log (N(i+1))-\log (N(i-1)))$. The local slope remains close to 2 at high temperatures for all sizes, but it approaches 1 for high sizes at low temperatures. In (a), the curves have been shifted vertically for clarity. The precise fits are the following: $\mathrm{T}=500 \mathrm{~K}: t_{e q}=810^{-12} N^{2.00}$; $\mathrm{T}=250 \mathrm{~K}: t_{e q}=810^{-9} N^{1.95} ; \mathrm{T}=125 \mathrm{~K}: t_{e q}=0.51 N^{1.52} ;$ $\mathrm{T}=100 \mathrm{~K}: t_{\text {eq }}=610^{3} N^{1.39} ; \mathrm{T}=83 \mathrm{~K}: t_{\text {eq }}=910^{7} N^{1.32}$ (b)

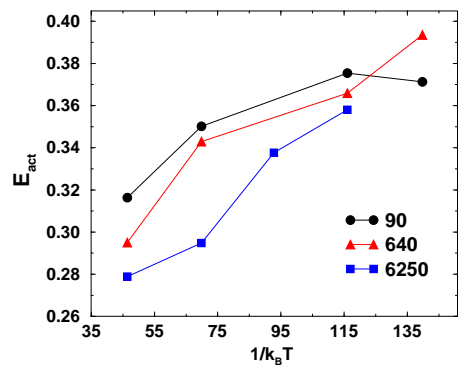

FIG. 6. (a) Time needed to reach equilibrium as a function of the temperature for different island sizes. (b) shows the local activation energy (defined as in Fig. 用) at each temperature.

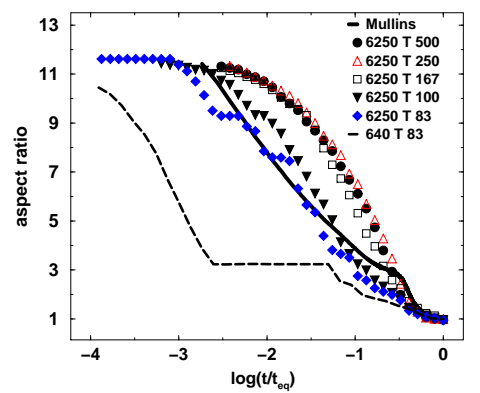

FIG. 7. Kinetic path followed by the islands as they relax to equilibrium. The solid line represents the prediction of Mullins' equation 2.8 and the different symbols to the relaxations obtained in the KMC simulations for islands containing 6250 atoms at the temperatures shown. The dashed line corresponds to a smaller island and illustrates the wide range of relaxation paths observed.

(a)

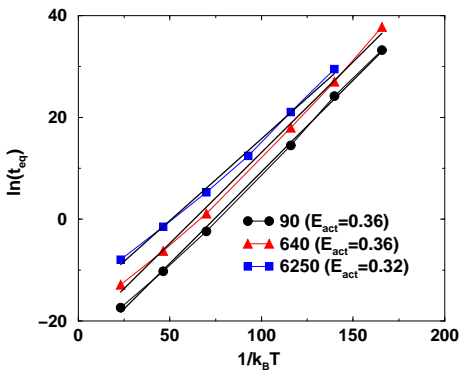

